\title{
SPHERICALLY SYMMETRIC T-SOLUTION OF THE EQUATIONS OF 5-DIMENSIONAL KALUZA-KLEIN THEORY
}

\author{
V.D. Gladush \\ Oles’ Honchar Dnipro National University, Dnipro, Ukraine \\ e-mailvgladush@gmail.com
}

\begin{abstract}
A geometrodynamical approach to the five-dimensional (5D) spherically symmetric cosmological model in the Kaluza-Klein theory is constructed. After dimensional reduction, the 5D Hilbert action is reduced to the Einstein form describing the gravitational, electromagnetic, and scalar interacting fields. The subsequent transition to the configuration space leads to the supermetric and the Einstein-HamiltonJacobi equation, with the help of which the trajectories in the configuration space are found. Then the evolutionary coordinate is restored, and the Cauchy problem is solved to find the time dependence of the metric and fields. The configuration corresponds to a cosmological model of the Kantovsky-Sachs type, which has a hypercylinder topology and includes scalar and electromagnetic fields with contact interaction. problem.

Keywords: action, Lagrange function, configuration space, Einstein-Hamilton-Jacobi equation, Cauchy
\end{abstract}

Received 12.10.2020; Received in revised form 15.11.2020; Accepted 01.12.2020

\section{Introduction}

The five-dimensional (5D) Kaluza-Klein (KK) theory in the traditional approach [1] is based on the following statements:

1. For a 5D metric $G_{A B}$ with signature $\{+,-,-,-,-\}$ of the space $\mathrm{M}^{5}$, the cylindricity condition is satisfied, according to which, it admits a spacelike Killing vector $\xi_{5}$. In the corresponding coordinate system, we have $\xi_{5}=\partial / \partial z$, which leads to the independence of the metric from the fifth coordinate $x^{4}=z$.

2. The condition that the space $\mathrm{M}^{5}$ is closed in the $z$-coordinate is satisfied, so that $0 \leq z \leq L$.

3. 4D physical quantities are introduced with the dimensional reduction of the $5 D$ theory.

This is done by the orthogonal 4+1-splitting of $\mathrm{M}^{5}$ and all geometric quantities in it, followed by the corresponding conformal mapping into the physical space-time $\mathrm{M}^{4}$.

There are other points of view on 5D gravity, among which we note the approach in which matter is induced by 5D geometry [2].

We follow the approach [1] and use the standard 5D Einstein-Hilbert action

$$
S=-\frac{1}{4 \pi l L} \int d^{5} x \sqrt{G}{ }^{(5)} R,
$$

where ${ }^{(5)} R$ is the 5D scalar curvature, $G=\operatorname{det}\left\|G_{A B}\right\|, d^{5} x=d x^{0} d x^{1} d x^{2} d x^{3} d x^{4}$. After integration over $z$ within the limits $0 \leq z \leq L$ and surface term neglection, it can be rewritten as follows

$$
S=-\frac{1}{4 \pi l} \int d^{4} x \sqrt{-h}\left[{ }^{(4)} R-\frac{1}{2} h^{\mu \nu} \psi_{, \mu} \psi_{, v}+\frac{1}{4} e^{-\sqrt{3} \psi} F_{\mu v} F^{\mu \nu}\right],
$$

5D metric has the form

$$
{ }^{(5)} d s^{2}=G_{A B} d x^{A} d x^{B}=e^{\psi / \sqrt{3}} d s^{2}-e^{-2 \psi / \sqrt{3}}\left(d z+A_{\mu} d x^{\mu}\right)^{2} .
$$

Here $d s^{2}=h_{\mu v} d x^{\mu} d x^{v}$ is the $4 \mathrm{D}$ physical metric, ${ }^{(4)} R$ is the scalar curvature of $\mathrm{M}^{4}$, 
$F_{\mu v}=A_{v, \mu}-A_{\mu, v}$ is the electromagnetic field tensor, $h=\operatorname{det}\left\|h_{\mu v}\right\|, \psi_{, \mu}=\partial \psi / \partial x^{\mu}, d^{4} x=d x^{0} d x^{1} d x^{2} d x^{3}$.

The resulting action has a natural physical meaning and describes a system of interacting scalar, electromagnetic, and gravitational fields. Moreover, in addition to the usual minimal interaction, it also contains the contact interaction of scalar and electromagnetic field characteristic of the 5D KK theory.

The solution of the equations that follow from action (2) was considered in many papers (see, for example, [3-5]).

\section{5D spherically symmetric T-model}

In the spherically symmetric case, the "T-solution" of the KK theory admits a spacelike Killing vector $\xi_{5}=\partial / \partial r$. Then the $5 \mathrm{D}$ and $4 \mathrm{D}$ metrics can be written as

$$
\begin{gathered}
{ }^{(5)} d s^{2}=e^{\psi / \sqrt{3}} d s^{2}-e^{-2 \psi / \sqrt{3}}\left(d z+\varphi d x^{0}\right)^{2}, \\
d s^{2}=f\left(x^{0}\right)\left(d x^{0}\right)^{2}-h\left(x^{0}\right) d r^{2}-R^{2}\left(x^{0}\right) d \sigma^{2} .
\end{gathered}
$$

Here all quantities depend only on the time coordinate $x^{0}$; in addition, we have $d \sigma^{2}=d \theta^{2}+\sin ^{2} \theta d \alpha^{2}$ and $E=F_{01}=A_{1,0}=\varphi_{, 0}$. Integrating over angles and over the coordinate $r$ within the limits $(-l / 2, l / 2)$, action (2), up to surface terms, can be rewritten as follows

$$
S=\int d x^{0}\left\{\frac{1}{\sqrt{f h}}\left[-2 R_{, 0}(R h)_{, 0}+\frac{1}{2} R^{2} e^{-\sqrt{3} \psi} \varphi_{, 0}^{2}\right]+\frac{1}{2} R^{2} \sqrt{\frac{h}{f}} \psi_{, 0}^{2}+2 \sqrt{f h}\right\} .
$$

To orthogonalize the action, we turn to the new field variables:

$$
f=N^{2} e^{-\lambda}, \quad h=e^{\lambda}, \quad R=e^{(\omega-\lambda) / 2} .
$$

Then the 4D metric and action (6) can be represented as

$$
\begin{gathered}
d s^{2}=e^{-\lambda}\left[N^{2}\left(d x^{0}\right)^{2}-e^{\omega} d \sigma^{2}\right]-e^{\lambda} d r^{2}, \\
S=\int L d x^{0}=\int\left(N^{-1} T+2 N\right) d x^{0}
\end{gathered}
$$

where $L$ is the Lagrangian of the configuration, $T$ is the kinetic part of the Lagrangian

$$
T=\frac{1}{2} e^{\omega}\left(\lambda_{, 0}^{2}-\omega_{, 0}^{2}+e^{-\lambda-\sqrt{3} \psi} \varphi_{, 0}^{2}+\psi_{, 0}^{2}\right) .
$$

Varying $S$ with respect to $N$, we obtain the constraint

$$
\frac{\delta L}{\delta N}=\frac{\partial L}{\partial N}=\frac{1}{2 c}\left\{-\frac{T}{N^{2}}+2\right\}=0 .
$$

Hence, it follows that $N=\sqrt{T / 2}$. This relation allows us to eliminate the factor $N$ from the Lagrangian $L$. As a result, we find that $L=2 \sqrt{2 T}$ and the action $S$ can be rewritten as follows

$$
S=2 \int \sqrt{2 T} d x^{0}=2 \int d x^{0} \sqrt{e^{\omega}\left(\lambda_{, 0}^{2}-\omega_{, 0}^{2}+e^{-\lambda-\sqrt{3} \psi} \varphi_{, 0}^{2}+\psi_{, 0}^{2}\right)} .
$$

Hence it can be seen that 


$$
S=2 \int \sqrt{e^{\omega}\left(d \lambda^{2}-d \omega^{2}+e^{-\lambda-\sqrt{3} \psi} d \varphi^{2}+d \psi^{2}\right)}=2 \int d \Omega
$$

Where

$$
d \Omega^{2}=e^{\omega}\left(d \lambda^{2}-d \omega^{2}+e^{-\lambda-\sqrt{3} \psi} d \varphi^{2}+d \psi^{2}\right)
$$

is the metric of the configuration space (CS) of field variables $\{\lambda, \omega, \varphi, \psi\}$.

Thus, from the initial action, we pass to the action of the system in the CS. It can be shown that geodesic equations that follow from the resulting configuration metric (14), together with the constraint, are equivalent to the Einstein equations. These geodesics can be found from the Einstein-Hamilton-Jacobi (EHJ) equation for the metric $\mathrm{d} \Omega^{2}(14)$ of the CS:

$$
\left(\frac{\partial S}{\partial \lambda}\right)^{2}-\left(\frac{\partial S}{\partial \omega}\right)^{2}+e^{\lambda+\sqrt{3} \psi}\left(\frac{\partial S}{\partial \varphi}\right)^{2}+\left(\frac{\partial S}{\partial \psi}\right)^{2}=4 e^{\omega}
$$

\section{Equations solution for the spherically symmetric T-model of the 5D KK theory}

After transforming field variables

$$
\xi=\lambda+\sqrt{3} \psi, \quad \eta=-\sqrt{3} \lambda+\psi
$$

the EHJ equation is transformed into an equation with separable variables. Its solution can be written as

$$
S=q \varphi+g \eta+\int d \omega \sqrt{a-4 e^{\omega}}+\frac{1}{2} \int d \xi \sqrt{a-4 g^{2}-q^{q} e^{\xi}}
$$

where $a$ is a separation constant, $q$ and $g$ are constants that have the meaning of electric and scalar charges. It is easy to see that only the case $a>4 g^{2}$ is possible.

For convenience, we introduce constants $m$ and $b$ using the formulas

$$
a=m^{2}+q^{2}+4 g^{2}>0, \quad b=a-4 g^{2}=m^{2}+q^{2}>0
$$

where the constant $m$ can be interpreted as a configuration mass. Note that the admissible range of the variables $\omega, \xi$ is determined by the inequalities $0<e^{\omega} \leq a / 4$ and $0<e^{\xi} \leq b / q^{2}$.

To find the trajectories, we differentiate the action by the parameters $\{q, g, a\}$ and equate the result with new constants:

$$
\left\{\begin{array}{l}
\frac{\partial S}{\partial q}=\varphi-\frac{q}{2} H(\xi)=C_{q}, \\
\frac{\partial S}{\partial g}=\eta-q g K(\xi)=C_{g}, \\
\frac{\partial S}{\partial a}=\frac{1}{2} M(\omega)+\frac{1}{4} K(\xi)=C_{a} .
\end{array}\right.
$$

Here $C_{q}, C_{g}, C_{a}$ are some constants, and the functions $M(\omega), K(\xi), H(\xi)$ are defined by the following integrals

$$
M(\omega)=\int \frac{d \omega}{\sqrt{a-4 e^{\omega}}}=\frac{1}{\sqrt{a}} \ln \frac{\sqrt{a}-\sqrt{a-4 e^{\omega}}}{\sqrt{a}+\sqrt{a-4 e^{\omega}}},
$$




$$
\begin{gathered}
K(\xi)=\int \frac{d \xi}{\sqrt{b-q^{2} e^{\xi}}}=\frac{1}{\sqrt{b}} \ln \frac{\sqrt{b}-\sqrt{b-q^{2} e^{\xi}}}{\sqrt{b}+\sqrt{b-q^{2} e^{\xi}}}, \\
H(\xi)=\int \frac{e^{\xi} d \xi}{\sqrt{b-q^{2} e^{\xi}}}=-\frac{2}{q^{2}} \sqrt{b-q^{2} e^{\xi}}
\end{gathered}
$$

This set of equations determines the trajectories of the system in the CS of field variables: $\{\omega, \xi, \varphi, \eta\}$. In order to find their dependence on time, it is necessary to reconstruct time as an evolutionary parameter $x^{0}$. After that, the Cauchy problem should be solved for the given initial conditions at the chosen initial moment of time. For this purpose, we first find the equation for the function $\omega=\omega\left(x^{0}\right)$ from the relations for the momentum conjugate to the variable $\omega$ :

$$
P_{\omega}=\frac{\partial L}{\partial \omega_{, 0}}=\frac{\partial S}{\partial \omega} .
$$

Hence, taking into account formulas (9), (10), and (16), we obtain the differential equation

$$
-\frac{1}{N} e^{\omega} \frac{d \omega}{d x^{0}}=\sqrt{a-4 e^{\omega}} .
$$

Choosing the calibration $N=1$, we find

$$
e^{\omega}=a / 4-\left(x^{0}\right)^{2}
$$

Moreover, we have $a / 4 \geq e^{\omega} \geq 0$, and $\left|x^{0}\right| \leq a / 2$. Substituting the expression for $e^{\omega}$ into the formula (20), we find

$$
M(\omega)=M(U)=\frac{1}{\sqrt{a}} \ln U
$$

where we introduce the notation

$$
0<U=\frac{\sqrt{a} / 2-\left|x^{0}\right|}{\sqrt{a} / 2+\left|x^{0}\right|}<1 .
$$

Now we can specify initial data. Bearing in mind the condition $\left|x^{0}\right| \leq a / 2$, it is natural to define the initial time as $x^{0}=0$. Therefore, we take the following initial conditions for the field variables $\left\{\lambda, \psi, \varphi, \xi, \eta, e^{\omega}\right\}$ at $x^{0}=0$ :

$$
\lambda_{0}, \psi_{0}, \varphi_{0}, \xi_{0}, \eta_{0}, e^{\omega_{0}}=a / 4
$$

Then formulas (20), (21), and (25)-(27) for $x^{0}=0$ imply

$$
\begin{gathered}
U\left(x^{0}\right)=1, \quad M\left(\omega_{0}\right)=M\left(x^{0}\right)=0 \\
\left\{\begin{array}{l}
K_{0}=K\left(\xi_{0}\right)=\frac{1}{\sqrt{b}} \ln \frac{\sqrt{b}-\sqrt{b-q^{2} e^{\xi_{0}}}}{\sqrt{b}+\sqrt{b-q^{2} e^{\xi_{0}}}}, \\
H_{0}=H\left(\xi_{0}\right)=-\frac{2}{q^{2}} \sqrt{b-q^{2} e^{\xi_{0}}} .
\end{array}\right.
\end{gathered}
$$


To construct a solution, it is convenient to rewrite the equation set (19) as follows

$$
\left\{\begin{array}{l}
\varphi-\frac{q}{2} H(\xi)=C_{q}, \\
\eta+4 g M(U)=C_{g}+8 g C_{a}, \\
2 M(\omega)+K(\xi)=4 C_{a} .
\end{array}\right.
$$

Then, taking into account the initial values (29), from here at $x^{0}=0$ we obtain

$$
\varphi_{0}-\frac{q}{2} H_{0}=C_{q}, \eta_{0}=C_{g}+8 g C_{a}, K_{0}=4 C_{a} .
$$

Using these relations, we exclude the unknown constants $C_{q}, C_{g}, C_{a}$ from the equations of motion (31). As a result, the equation system (31) takes the form

$$
\left\{\begin{array}{l}
\varphi-\varphi_{0}=\frac{q}{2}\left(H(\xi)-H_{0}\right), \\
\eta-\eta_{0}=-4 g M(U), \\
2 M(\omega)=K_{0}-K(\xi) .
\end{array}\right.
$$

Taking into account formulas (21), (22), and (26), as well as initial values (30), this system can be rewritten as follows

$$
\left\{\begin{array}{l}
\varphi-\varphi_{0}=-\frac{1}{q}\left(\sqrt{b-q^{2} e^{\xi}}-\sqrt{b-q^{2} e^{\xi_{0}}}\right), \\
\eta-\eta_{0}=-\frac{4 g}{\sqrt{a}} \ln U, \\
\ln U^{p}=\ln \frac{\sqrt{b}-\sqrt{b-q^{2} e^{\xi_{0}}}}{\sqrt{b}+\sqrt{b-q^{2} e^{\xi_{0}}}}-\ln \frac{\sqrt{b}-\sqrt{b-q^{2} e^{\xi}}}{\sqrt{b}+\sqrt{b-q^{2} e^{\xi}}}
\end{array}\right.
$$

where $p=2 b / a$. Next, we express the quantities $\varphi$ and $e^{\xi}$ through the function $U$. For this purpose, we rewrite the last equation in the system (34) as follows

$$
\frac{\sqrt{b}-\sqrt{b-q^{2} e^{\xi}}}{\sqrt{b}+\sqrt{b-q^{2} e^{\xi}}}=V \text {. }
$$

Here we introduce the notations

$$
V=\frac{1-B}{1+B} U^{-p}, \quad B=\sqrt{1-\frac{q^{2}}{b} e^{\xi_{0}}}<1 .
$$

Note that, by virtue of the inequality $0<U<1$, for admissible values of $\xi_{0}\left(-\infty<\xi_{0}<\right.$ $<\ln \left(b / q^{2}\right)$ it follows that $0<V<1$. Solving equation (35) with respect to $e^{\xi}$, we obtain

$$
e^{\xi}=\frac{4 b}{q^{2}} \frac{V}{(1+V)^{2}}=\frac{4 b}{q^{2}}\left(V^{-1 / 2}+V^{1 / 2}\right) .
$$

Substituting the expression for $V$ from (36) into the formula (37), we find 


$$
e^{\xi}=\left\{\frac{q}{2 B}\left[\left(\frac{1-B}{1+B}\right)^{-1 / 2} U^{p / 2}+\left(\frac{1-B}{1+B}\right)^{1 / 2} U^{-p / 2}\right]\right\}^{-2} .
$$

Further, taking into account the relations

$$
\frac{1-B}{1+B}=\frac{b}{q^{2}}(1-B)^{2} e^{-\xi_{0}}=\frac{q^{2}}{b} \frac{e^{\xi_{0}}}{(1+B)^{2}},
$$

the expression (38) for $e^{\xi}$ can be rewritten as follows

$$
e^{\xi}=e^{\xi_{0}}\left[\left(\frac{1+B}{2}\right) U^{p / 2}+\left(\frac{1-B}{2}\right) U^{-p / 2}\right] .
$$

Let us now find the potential of the electric field $\varphi$. Substituting the expression for $e^{\xi}$ from formula (37) into the first equation of the system (34) and taking into account (36), we find

$$
\varphi-\varphi_{0}=\frac{q}{\sqrt{b}}\left(\frac{1-V}{1+V}-B\right) .
$$

Finally, substituting $V$ and $B$ from relations (36) into this formula, we obtain

$$
\varphi-\varphi_{0}=\frac{q}{\sqrt{b}} \frac{\left(1-U^{p}\right) e^{\xi_{0}}}{1-B+(1+B) U^{p}} .
$$

Thus, relations (25), (26), (40), (41), and (34) for $\eta$ represent the desired solution to the Cauchy problem with initial data (28) and determine the evolution of the spherically symmetric 5D T-configuration in terms of the field variables $\{\omega, \xi, \varphi, \eta\}$.

\section{Conclusions}

The paper demonstrates the effectiveness of the Hamilton-Jacobi method in finding solutions to the Einstein equations for systems with one evolutionary coordinate and particularly for constructing spherically symmetric T-configurations of the equations of 5D KK theory with a degenerate Lagrangian. In the CS, geodesics correspond to the required configurations. The direct solution of the EHJ equation leads to geodesics in an implicit form. Along the way, with finding the CS supermetric, the differential-geometric structure of the CS is clarified, its vectors and Killing tensors and the corresponding conservation laws are found, and the CS curvature tensor is calculated. The EHJ method for T-configurations has its own peculiarities. When we transit from CS to the coordinate space, it becomes necessary to restore time. For this, the Cauchy problem is solved with initial data on the hypercylinder of an initial radius.

\section{References}

1. Vladimirov, Yu.S. Klassicheskaya teoriya gravitatsii / Yu.S. Vladimirov. - M.: Knizhnyy dom Librokom, 2009. - 264 p.

2. Wesson, P.S. Space, Time, Matter. Modern Kaluza-Klein Theory / P.S. Wesson Singapore: World Scientific, 2000. $-217 \mathrm{p}$.

3. Bronnikov, K.A. O vzaimodeystvuyushchih poliah $\mathrm{v}$ obshchey teorii otnositel'nosti / K.A. Bronnikov, G.Ya. Shikin // Izvestiya vuzov SSSR. Fizika, - 1977. - No. 9 - P. 25-30.

4. Gladush, V.D. Static spherically symmetric solution for interacting scalar, electromagnetic and gravitational fields / V.D. Gladush // Sov. Phys. J. - 1980. - Vol. 23, No. 3. - P. 240-246.

5. Bronnikov, K.A. Modeli samogravitiruyushchih chastits s klassicheskimi poliami i ih ustoychivost' / K.A. Bronnikov, G.Ya. Shikin // Itogi nauki i tehniki. Ser. Klassicheskaya teoriya polia i teoriya gravitatsii. T. 2: Gravitatsiya i kosmologiya. - M.: VINITI, 1991. - P. 4-55. 